\title{
Access-Oriented Energy Storage Configuration Method to Stabilize Fluctuation of Pulsed Load
}

\author{
Zhu Hong ${ }^{1}$, Bian Haihong ${ }^{*}, 2$ and Ji Wenlu ${ }^{1}$ \\ ${ }^{I}$ State Grid Nanjing Power Supply Company, Nanjing, Jiangsu, 210008, P.R. China \\ ${ }^{2}$ School of Power Engineering, Nanjing Institute of Technology, Nanjing, 211167, P.R. China
}

\begin{abstract}
It is necessary to minimize the negative influence caused by pulsed load on microgrid. By analyzing impulse load characteristic, access-oriented energy storage, especially super capacitor, turned out to be suitable energy storage to stabilize this huge fluctuation. This paper presents an optimal configuration method of energy storage system (ESS) in microgrid, which is based on the result of spectrum analysis of sample data of pulsed load. This method both takes into consideration the stability of system and energy storage cost at the same time. The effectiveness of this approach was verified by case analysis of a typical steel plant with pulsed load using MATLAB software.
\end{abstract}

Keywords: Super capacitor, rated power and capacity, pulsed load, spectrum analysis.

\section{INTRODUCTION}

Recently microgrid has raised public's attention due to its stability, flexibility and short-term response. A microgrid is defined as a small-scale intelligent power network which includes at least one load and one distributed energy resource. The microgrid is regarded as a controllable load from the system operator's point of view as it would supply its own load and respond to real-time electricity price variations. By microgrid implementation, the cost of supplying energy is lowered, local reliability and power quality is improved, and system emission is reduced. Despite its huge potential and fast development, there are still many problems existing [1]. With the expansion of industrial production scale, large capacity fluctuating loads have gradually emerged, which set higher requirement of tolerance capacity and stability of grid. As a consequence of its inherent characteristics such as low inertia and weak damping, microgrid lacks the ability of disturbance resistance at the moment of load demand changing [2]. Thus ESS is of great importance for microgrid to maintain energy balance, especially for the one with pulsed load.

Research on influence of pulsed load on grid has already begun [3]. We analyzed the effect of pulsed load from heavy steel rolling mill on grid frequency [4]. We calculated the voltage fluctuation produced by ultra-high power electric arc furnace through allocating reactive power compensation device [5]. We reduced power fluctuation by propertiesbased control mode [6]. We proposed a new method which analyzed influence of pulsed load of steel plant on grid considering time uncertainty. Additionally, research on ESS configuration has already made some achievements [7]. We put forward an ESS configuration method based on saturation control theory [8]. We studied the configuration

*Address correspondence to this author at the Nanjing Institute of Technology, Nanjing, P.R. China; Tel: +86 13655183302;

E-mail: bhh_njit@126.com method applied to peak load shifting and made the economic assessment [9]. Later, we presented estimation of wind farm energy storage capacity for short-term power dispatch.

In sum, at present domestic research on pulsed load is mainly for large power system and research on ESS configuration is mainly for wind/photovoltaic hybrid power system. There are few researches on ESS configuration method for pulsed load.

In this essay, super capacitor is utilized as energy storage device according to the special characteristic of pulsed load. The optimization objective is to minimize the energy storage capacity.

\section{ENERGY SYSTEM CONFIGURATION}

\subsection{Pulsed Load Characteristics}

Pulsed load mainly comes from steel machine and arc furnace and will produce large harmonic current and power fluctuation, which usually causes frequency surging and voltage flicker. Due to the fierce change of reactive power, the rapid fluctuation of voltage near point of common coupling (PCC) will occur, which has seriously negative effect on the operation of electrical equipment such as transformer and voltage transformer (PT) [10], especially in the term of acceleration of insulation aging, occurrence of units oscillation, increase of equipment forced outage and lifespan shortening. Additionally, pulsed load is non-line load whose interaction with grid cannot be described by simple linear superposition [11][12]. Thus the influence scope of pulsed load cannot be estimated directly.

The characteristics of pulsed load are shown as follow: (1) large varied amplitude. Its peak may be several times of average load; (2) quick change. But the time of peak occurrence is short and the load power is in smooth and stable state most of time; (3) random change. But the varying pattern of pulsed load is basically the same for a certain type of load. 
Table 1. Comparison between types of energy storage.

\begin{tabular}{|c|c|c|c|c|c|}
\hline Type & $\begin{array}{c}\text { Energy Density } \\
\text { (Wh/kg) }\end{array}$ & $\begin{array}{c}\text { Power Density } \\
\text { (W/kg) }\end{array}$ & Life Span (year) & Efficiency (\%) & Annual Cost (¥/kWh) \\
\hline \hline Super Capacitor & $2-5$ & $7000-18000$ & 30 & 95 & 750 \\
\hline SMES & $<1$ & 1000 & 30 & 90 & 1800 \\
\hline Lead-acid Battery & $30-200$ & $100-700$ & 8 & 90 & 120 \\
\hline Flywheel & $5-50$ & $180-1800$ & 30 & & 500 \\
\hline
\end{tabular}

It can be seen from above characteristics that the key to reduce the influence of pulsed load is to mitigate the power fluctuation [13]. In this paper, energy storage system is equipped with microgrid to shift the pulsed load and maintain the power volatility within the admitted scope, which reduce the impact to the microgrid.

\subsection{Storage}

Energy storage device is one of the indispensable components for microgrid transient stability. With its charge/discharge characteristic, it can store surplus energy and release energy when load demand increases. Furthermore, with the support of energy storage, it would be possible to realize control improvement, volatility mitigation, load following, voltage stability, frequency stability, load management and power quality improvement. An accurate and practical ESS model would increase modeling of microgrid operation from both economic and security perspectives [14]-[16].

The optimal size of ESS including rated power and rated capacity is of vital importance in a microgrid as small ESS may not offer the objectives of economic benefits, desired flexibility or predefined reliability in the microgrid and the large ESS increases the cost of investment and maintenance costs. Therefore, ESS needs to be optimally sized in order to balance the economic aspect and the requirement of system reliability [17]-[19].

At present, energy storage technology mainly include: fly wheel, super capacitor, SMES, battery [20]. Each type has its own characteristics and range of application, which is shown in Table 1 [21].

The main factors influencing the energy storage system configuration are the characteristics of load, the reaction rate, round-trip efficiency and lifespan. Actually when choosing the type of energy storage, all above factors should be considered comprehensively for a better result.

At present, wind/photovoltaic hybrid microgrid usually adopts battery as energy storage because of its high energy density which can satisfy the energy demand of peak load. However it does not apply to pulsed load because it has little tolerance of high charge voltage and high charge current. Moreover it also lacks the ability of quick shift between charge and discharge [22].

As is also shown in the Table 1, both flywheel and SMES have lower round-trip efficiency and power density than super capacitor. What's more, it takes about 1 hour to charge flywheel four times, which cannot satisfy the requirements of pulsed load characteristics of random and frequent charging. Additionally the investment of SMES is much higher than others so it has not been in its large-scale application.

By contrast, super capacity has advantages in high power density and fast charging rate so that it can offer fast access to power whenever needed, which can satisfy the frequent high power charging and discharging for pulsed load. On the other hand, although the instantaneous power is high, its energy fluctuation is not fierce because of its short duration. As a consequence, the required energy is comparatively small matching its low power density [23][24]. Table 2 gives a classification of different power generation technologies in terms of access and capacity orientation.

Table 2. Classification of energy storage.

\begin{tabular}{|c|c|}
\hline Storage Class & Storage Technology \\
\hline \hline & Super Capacitor \\
Access-oriented & Flywheel \\
& SMES \\
& High-power Battery \\
\hline Capacity-oriented & High-energy Battery \\
& Hydrogen Energy Storage \\
\hline
\end{tabular}

To sum up, super capacitor is one of the suitable energy storage devices for reducing the fluctuation of pulsed load in microgrid.

\subsection{Modeling}

In this part, an ESS configuration model is established to determine the optimal ESS energy capacity for smoothing the pulsed load power fluctuation. Firstly make spectrum analysis [25] [26] of pulsed load sampling data. And then compensated frequency band which satisfies the fluctuation requirement and constraints of state of charge (SOC) and continuous operation can be determined based on the result. Finally the rated power and capacity of ESS can be obtained.

\subsubsection{Control Objective}

To assess the compensation effect of ESS, maximum fluctuation rate (MFR) within time period $T_{e}$ should be calculated. The index can be used to illustrate the relative value of fluctuation in the specific time-window. Therefore, choosing appropriate reference value seems more significant. 
Supposing the reference value, $P_{N}$, means the average of all sample amplitudes, the MFR can be calculated as below:

$F_{T_{\mathrm{e}}}=\frac{P_{T_{\mathrm{e}}}^{\max }-P_{T_{\mathrm{e}}}^{\min }}{P_{\mathrm{N}}} \times 100 \%$

where

$P_{T_{\mathrm{e}}}^{\max }$---the maximum power within $T_{e}$

$P_{T_{\mathrm{e}}}^{\min }$---the minimum power within $T_{e}$

The objective of the ESS installation concentrates on declining $F_{T_{e}}$ to a certain extent to satisfy the requirement of power fluctuation.

\subsubsection{Constraints}

According to the inherent characteristic and economic operation requirement, ESS should satisfy the following constraints in practical operation.

1) State of charge constraint

$S O C_{\text {low }} \leq S O C \leq S O C_{u p}$

where,

$S O C_{u p}$--- the upper limit of SOC;

$S O C_{\text {low }}$--- the lower limit of SOC.

Both upper limit and lower limit of SOC range from 0 to 1. SOC reflects remain energy of ESS. It defines as the percentage of remain energy in the total capacity. The overcharge can seriously affect the lifespan of energy storage device so it is a must to keep SOC in a proper range.

2) Continuous operation constraint

$\Delta E=\sum_{n=1}^{N_{s}}\left(\frac{P_{b}(i) T_{s}}{3600}\right)=0$

where,

$T_{s}$--- the sampling period;

$\Delta E$--- the net charge/discharge energy;

$P_{b}$--- the ESS output

After ESS compensation the power pattern should not only meet the requirement of control objective, but also ensure ESS can operate continuously. As a result of this, ESS net charge and discharge electricity need to be nearly equal in the whole sampling period.

\section{Rated power}

The maximum ESS charging power satisfying the objective output power can be acquired by Discrete Fourier Transform (DFT). This is calculated as follows:

$\left\{\begin{array}{l}A_{g}=F(\Delta(t))=\left[A_{g}(1), \ldots, A_{g}(i), \ldots, A_{g}\left(N_{s}\right)\right]^{T} \\ f_{g}=\left[f_{g}(1), \ldots, f_{g}(i), \ldots, f_{g}\left(N_{s}\right)\right]^{T}\end{array}\right.$ where,

$\Delta(t)$--- the time series of power sample;

$A_{g}$---the amplitude vector of the Fourier transformation;

$f_{g}$---the frequency vector of the Fourier transformation;

$R_{g}$---the real part of $A_{g}$ (in frequency domain);

$I_{g}$--- the imaginary part of $A_{g}$ (in frequency domain).

Using DFT, $f_{g}(i)$ is calculated below:

$f_{g}(n)=\frac{f_{s}(n-1)}{N_{s}}=\frac{n-1}{T_{s} N_{s}}$

where,

$f_{s}$--- the sampling frequency $(\mathrm{Hz})$.

As a result, the complex vector $A_{g}$ can be represented on horizontal axis, which is labelled as a fraction of sampling rate between DC and one-half of the sampling rate.

We can acquire the smoothed target output by calculating Inverse Discrete Fourier Transform (IDFT) of $A_{g}$ from (6) and (7).

$A_{0}(n)=\left\{\begin{array}{cc}0+j 0, & f_{i} \in \boldsymbol{F}_{\mathrm{ps} 1} \cup \boldsymbol{F}_{\mathrm{ps} 2} \\ A_{g}(i), & f_{i} \notin \boldsymbol{F}_{\mathrm{ps} 1} \cup \boldsymbol{F}_{\mathrm{ps} 2}\end{array}\right.$

$\boldsymbol{P}_{0}=F^{-1}\left(\boldsymbol{A}_{0}\right)=\left[P_{0}(1), \ldots, P_{0}(i), \ldots, P_{0}\left(N_{s}\right)\right]^{\mathrm{T}}$

where,

$\boldsymbol{F}_{\mathrm{ps} 1}$--- the range of determined compensation frequency;

$\boldsymbol{F}_{\mathrm{ps} 2}---$ the range in Nyquist frequency.

From equation (4), for the range between compensation frequency and Nyquist frequency, amplitudes of the components are set to zero, while amplitudes outside this range remain the same. In (7), $F^{-1}$ indicates IDFT.

Because of the smoothed output $P_{0}$ derivation, the ESS output can be easily calculated as:

$P_{b 0}(i)=P_{0}(i)-P_{\mathrm{g}}(i)$

When $P_{b 0}(i)$ is positive, it means that the ESS should discharge; when $P_{b 0}(i)$ is negative, it means that the ESS should be charged. Given the storage efficiency of ESS, the factual output power can be calculated as (9).

$P_{\mathrm{ESS}}(n)=\left\{\begin{array}{cc}\frac{P_{b 0}(n)}{\eta_{\mathrm{dc}}}, & P_{b 0}(n) \geq 0 \\ P_{b 0}(n) \eta_{\mathrm{c}}, & P_{b 0}(n)<0\end{array}\right.$

where. 
$\eta_{\mathrm{dc}}$--- the discharge efficiency of ESS;

$\eta_{\mathrm{c}}$--- the charge efficiency of ESS.

\section{Rated capacity}

In (10), a time series storage capacity of ESS can be performed by the integration of the ESS output power.

$$
E(m)=\sum_{0}^{m}\left(P_{b}(m) \frac{T_{\mathrm{s}}}{3600}\right), \quad m=0,1, \ldots, N_{s}
$$

Furthermore another significant point is to maintain the energy balance during the operating cycle (the sample period) when the charge and discharge energy of the ESS should be approximately zero. Otherwise a revision has to be made to the smoothed $P_{0}$ to recompense storage losses. The ESS compensation frequency can be identified to minimize target output power $P_{0}$.

Given the capacity constraints and the storage level $E(m)$, the capacity configuration of ESS is obtained as

$E_{\mathrm{ESS}}=\frac{\max E(m)-\min E(m)}{S O C_{\mathrm{up}}-S O C_{\mathrm{low}}}$

As to the ideal energy storage device, $S O C_{\text {up }}$ and $S O C_{\text {low }}$ are 1 and 0 respectively. However both overcharge and overwork should be avoided in the actual operation circle, which requires the SOC within a proper range. Obviously $\max E(m)-\min E(m)$ represents the maximum energy variation in the whole operating period.

\section{SIMULATION AND VALIDATION}

The simulation analyses were performed based on the pulsed load data of a steel plant in Jiangsu Province and then determine the rated power and capacity of ESS and discuss the economic benefit of super capacitor. In order to make optimal results more visual, only one rolling machine's data was analyzed. Additionally what should to be point out is that when several machines run at the same time, the fluctuation is more violent and random. But the processing method is the same.

According to the charging characteristic of super capacitor, set charge and discharge efficiency equal and both are $97.47 \%$; the upper limit and lower limit are 0.9 and 0.5 respectively. The control objective is to realize the final power fluctuation rate within $10 \%$. The graph of the initial impact power shows as Fig. (1).

Firstly process the data by DFT and then adopt trial and error calculation to obtain its amplitude-frequency characteristic, based on which the objective ESS frequency band for compensation can be determined. The period range is $[2,170.7]$ (s) corresponding to its frequency band. Notice that if the frequency band for compensation is longer, the fluctuation of ESS output power will be fiercer and the charge/discharge frequency will be higher.

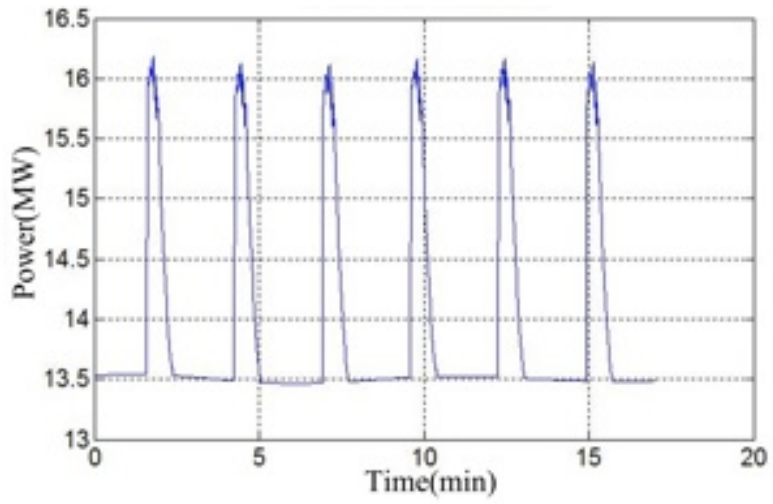

Fig. (1), Actual power of pulsed load.

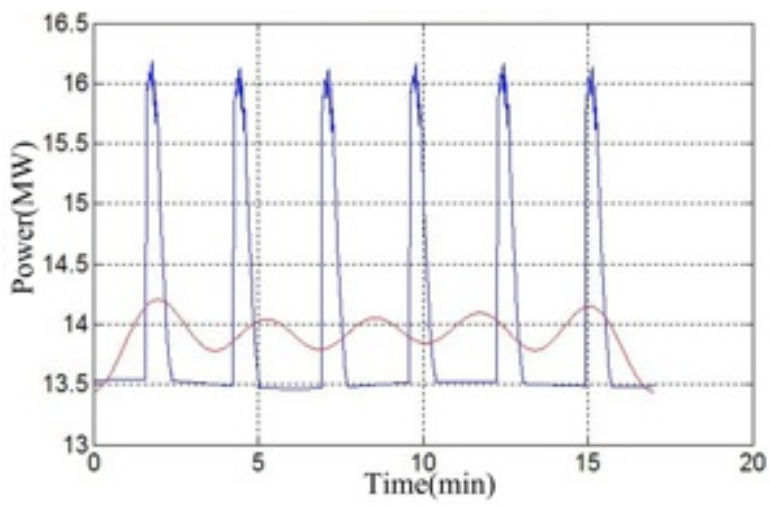

Fig. (2), Initial pulsed power and compensated power.

Next handle the compensated frequency spectrum by Inverse Discrete Fourier Transform (IDFT). Considering the loss of charge/discharge, shift the power line upward and acquire the objective power output line with gentle fluctuation implying that pulsed load has been compensated to a great extent, as is shown in Fig. (2).

The actual ESS output power, the fluctuation of energy and SOC are shown as Figs. (3-5) respectively. It is easy to find that ESS is with short-time response and both power fluctuation and SOC can satisfy constraints above.

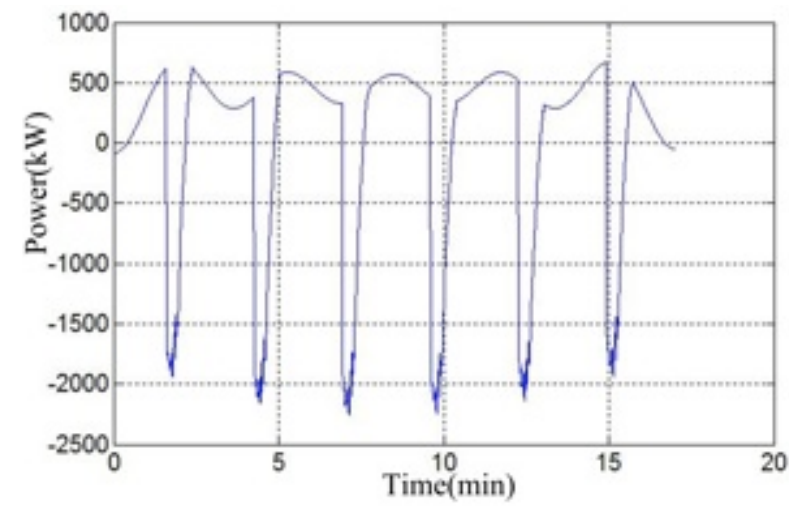

Fig. (3), Actual ESS output power.

By analyzing, the load need to be equipped with ESS whose rated capacity is $42.65 \mathrm{~kW}$ and rated power is $2.256 \mathrm{MW}$. Notice that power density is larger than energy density, which corresponds to the analysis above. Table 1 can be used to calculate the gross cost of the super capacitor (about $500 ¥$ per year) while the cost of battery is much 
higher (about $77000 ¥$ per year). It can be concluded that by adopting super capacitor, ESS can not only have quick response but also realize the reduction of the invention and operation cost.

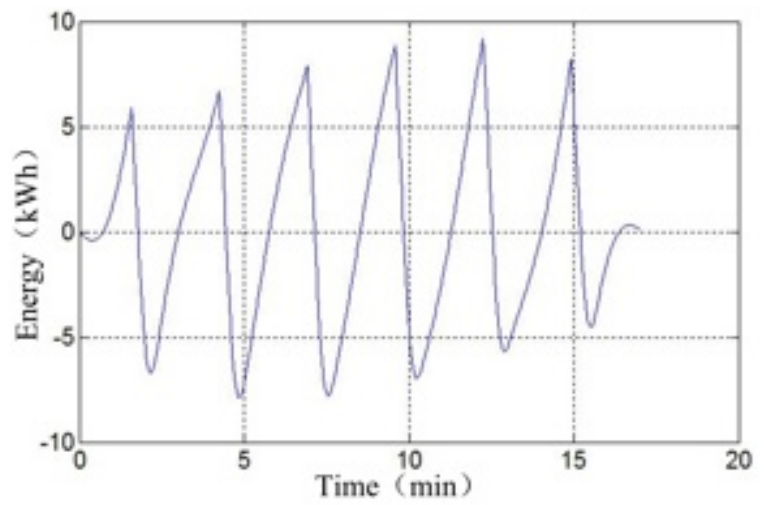

Fig. (4), Fluctuation of ESS energy.

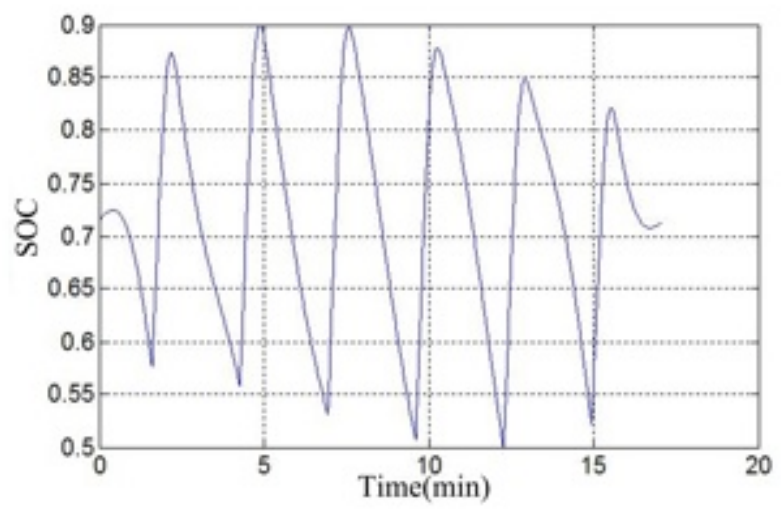

Fig. (5), Fluctuation of SOC.

\section{CONCLUSION}

In order to reduce the negative effect of pulsed load on microgrid and increase the stability and resistance of disturbance, this paper proposed an approach to mitigate the impact by ESS and further pointed out ESS configuration method to determine the rated power and capacity. This method can both satisfy the required fluctuation rate and constraints of SOC and continuous operation so that the compensated power pattern is comparatively smooth. The negative effect of pulsed load is mitigated consequently.

Case analysis in the last section indicates that the proposed method is effective and with comparatively low cost. Furthermore it takes into consideration the efficiency of charge and discharge, potential pollution and ESS lifespan.

Additionally, considering the advantages and disadvantages of battery and super capacitor; battery is high energy density and has slow response while super capacitor is high power density and has quick response; hybrid ESS with combination of these two types can be utilized in order to integrate the advantages of each other for a better consequence.

From the above-mentioned content, centrifugal extruder, a new polymer processing machine, is provided. And by the mathematical analysis and experiments, it is shown that the centrifugal force field can provide the solid-plug conveying pressure sufficiently and stably, which can prove the industrial practicability of centrifugal extruder. And the temperature distribution of solid-plug conveying phase is achieved in which the rotor speed affects the temperature of the rotor surface, as the faster the speed, the lower the temperature.

\section{CONFLICT OF INTEREST}

The author confirms that this article content has no conflict of interest.

\section{ACKNOWLEDGEMENTS}

This work was financially supported by the Natural Science Foundation of Jiangsu Province (BK20130742), the Nanjing Institute of Technology Fund(YKJ201316).

\section{REFERENCES}

[1] S. Bahramirad and H. Daneshi,"Optimal sizing of smart grid SMSTM storage management system in microgrid," in Proc. Innov. Smart Grid Technol. Conf. (ISGT), Washington, DC, Jan. 2012

[2] W. Liu, H. Yang and B. Zhu, "Survey on key technologies of microgrid," Power System Protection and Control, vol. 40, no. 14 pp. 152-155, Jul. 2012. (in Chinese)

[3] L. Li, W. Huang and X. Ma. "Application of super capacitor energy storage in micro-grid," Shanxi Electric Power, vol. 38, no. 8, pp. 12-16, 2012. (in Chinese)

[4] G.L. Chen, "Study on SVC digital regulator for industrial load,"B.S.thesis, Xi an University of Technology, Xi an, Shanxi, China, 2007. (in Chinese)

[5] J.M. Crider and S. D. Scott, "Reducing impact of pulsed power loads on microgrid power systems," IEEE Transactions on Smart Grid, vol. 1, no. 3, pp. 270-277, Dec. 2010.

[6] Y. Hu, Z. Chen, Z. Chen and Y. Yuan. "A new method for analyzing the influence of the impact load in steel plant on grid," in Proc. 2011 4th International Conference on Electric Utility Deregulation and Restructuring and Power Technologies (DRPT), Weihai, China, 2011, pp. 241-244.

[7] Y. Wu, Y. Sun, J. Xu, et al. "Determination methodology for energy storage capacity based on saturation control theory," Proceedings of the CSEE, vol.31, no. 22, pp. 32-39, Aug. 2011. (in Chinese)

[8] X. Xiu, J. Li and D. Hui, "Capacity configuration and economic evaluation of energy storage system for grid peak load shifting," Electric Power Construction, vol. 34, no. 2, pp. 1-5, Feb. 2013. (in Chinese)

[9] F. Kong, Q. Chao, F. Yuan, et al. "Estimation of wind farm energy storage capacity for short term power dispatch," Electric Power Automation Equipment, vol. 32, no. 7, pp. 21-24, Jul. 2012. (in Chinese)

[10] H. A. Smolleck, R. Satish, N.R. Prasad, R. O. Velasco, "Effects of pulsed-power loads upon an electric power grid," IEEE Transactions on Power Delivery, vol. 6, no. 4, pp. 1629-1640, Nov. 1991.

[11] J. Wang, W. Guo and Z. Bie, "Influence of shock loads on power system reliability," Processings of the CSEE, vol. 31, no. 10, pp. 59-64, Apr. 2011. (in Chinese)

[12] H. Beng, S. Zou, Y. Fu, et al. "Research of the power quality problem and treatment scheme for impact loads connected into," Power System Protection and Control, vol. 42, no. 1, pp. 54-61, Jan. 2014. (in Chinese)

[13] X. Tang, Z. Qi, "Energy storage technologies and control methods of micro grid," Acts Energiae Solaris Sinica, vol. 33, no. 3, pp. 517-524, Mar. 2012. (in Chinese)

[14] A. Joseph and M. Shahidehpour, "Battery storage systems in electric power systems," in Proc. IEEE Power Energy Soc. Gen. Meet., 2006.

[15] M. K. C.Marwali, H. Ma, M. Shahidehpour, and K.H. AbdulRahman, "Short term generation scheduling in photovoltaic-utility 
grid with battery storage," IEEE Trans. Power Syst., vol. 13, no. 3, pp. 1057-1062, Aug. 1998.

[16] M. Shahidehpour, "Role of smartmicrogrid in a perfect power system," in Proc. IEEE Power Energy Soc. Gen. Meet., 2010.

[17] X.Wang, D.M. Vilathgamuwa, and S. Choi, "Determination of battery storage capacity in energy buffer for wind farm," IEEE Trans. Energy Convers., vol. 23, no. 3, pp. 868-878, Sep. 2008.

[18] S. Chiang, K. Chang, and C. Yen, "Residential photovoltaic energy storage system," IEEE Trans. Energy Convers., vol. 45, no. 3, pp. 385-394, Jun. 1998.

[19] C. Venu, Y. Riffonneau, S. Bacha, and Y. Baghzouz, "Battery storage system sizing in distribution feeders with distributed photovoltaic systems," in Proc. IEEE Bucharest PowerTech, Jun. 2009.

[20] P. F. Ribeiro, B. K. Johnson, M. L. Crow, A. Arsoy, "Energy storage systems for advanced power applications," Proceedings of the IEEE, vol. 89, no. 12, pp. 1744-1756, Dec. 2001.

[21] L. Zhou, Y. Huang, K. Guo, et al. "A survey of energy storage technology for micro grid," Power System Protection and Control, vol. 39, no. 7, pp. 147-152, Apr. 2011. (in Chinese)
[22] Y. V. Makarov, P. Du, M. C. W. Kintner-Meyer, C. Jin, H. F. Illian, "Sizing energy storage to accommodate high penetration of variable energy resources," IEEE Transactions on sustainable Energy, vol. 3, no. 1, pp. 34-40, Jan. 2012.

[23] W. Huang, X. Wang, J. Guo, et al. "Discussion on application of super capacitor energy storage system in microgrid," in Proc. International Conference on Sustainable Power Generation and Supply, Nanjing, China, 2009, pp. 1-4.

[24] P. K. Soori, S. C. Shetty, S. Chacko, "Application of super capacitor energy storage in microgrid system," in Proc. 2011 IEEE GCC Conference and Exhibition. Dubai, United Arab Emirates, 2011, pp. 581-584.

[25] C. Wang, B. Yu, J. Xiao, et al. "Sizing of energy storage system in microgrid for stabilization of tie-line power flow," Automation of Electric Power Systems, vol. 37, no. 3, pp. 12-17, Sep. 2013. (in Chinese)

[26] B. Chen, Q. Li, X. Yuan, et al. "Influences and Analysis of Large Impact Loads on Power Quality of Grid," in Proc. 2011 4th International Conference on Electric Utility Deregulation and Restructuring and Power Technologies (DRPT). Weihai, China, 2011, pp. 1282-1289.

Received: May 26, 2015

Revised: July 14, 2015

Accepted: August 10, 2015

(C) Hong et al.; Licensee Bentham Open.

This is an open access article licensed under the terms of the Creative Commons Attribution Non-Commercial License (http://creativecommons.org/licenses/by$\mathrm{nc} / 3.0 /$ ) which permits unrestricted, non-commercial use, distribution and reproduction in any medium, provided the work is properly cited. 